\title{
Conceptos y esquemas en la argumentación.
}

\section{Concepts and schemes in argumentation.}

\author{
Fabián Bernache Maldonado \\ Departamento de Filosofía / Universidad de Guadalajara (MÉXICO) \\ CE: fabian.bernache@csh.udg.mx ID ORCID: 0000-0001-7158-892X
}

DOI: $\underline{10.32870 / \text { sincronia.axxiii.n76.6b19 }}$

Esta obra está bajo una Licencia Creative Commons Atribución-NoComercial 4.0 Internacional

Recibido: 06/02/2019

Revisado: 21/02/2019

Aprobado: 19/03/2019

\section{RESUMEN}

En este trabajo, sostenemos la idea de que nuestra capacidad de formar juicios es el factor principal que explica nuestro dominio de la dimensión lógica de la argumentación, es decir nuestra capacidad de identificar razones relevantes para respaldar nuestras aserciones o para refutar las aserciones de un interlocutor con el que estamos en desacuerdo. La capacidad de formar juicios presupone la sensibilidad a las restricciones o normas racionales que regulan la aplicación de nuestros conceptos y es esta forma de sensibilidad lo que nos permite identificar buenas razones para respaldar o refutar aserciones. Nuestra propuesta es comparada con la influyente propuesta de los esquemas de argumentación, principalmente defendida por Douglas Walton. El propósito de esta comparación es mostrar las ventajas de nuestra propuesta, pues no solamente se trata de un planteamiento más simple, sino que, a partir de esta propuesta, es posible también explicar el funcionamiento y la racionalidad de los esquemas de argumentación de Walton y resolver algunas de sus insatisfacciones.

Palabras clave: Razones. Juicios. Argumentos. Aplicación de conceptos. Cognición. 


\begin{abstract}
:
In this work, we claim that our capacity to form judgments is the main factor that explains our practical knowledge of the logical dimension of argumentation, that is our capacity to identify relevant reasons that are able to support our assertions or to refute the assertions of an interlocutor with whom we disagree. The capacity to form judgments presupposes a kind of sensibility to the rational restrictions or norms that regulate the application of our concepts and it is this kind of sensibility that enables us to identify good reasons in order to support or refute assertions. Our proposal is compared with the influential proposal of argumentation schemes, mainly defended by Douglas Walton. The aim of this comparative strategy is to show the advantages of our proposal, for we claim that it is not only more simple, but that it enables also to explain the functioning and rationality of Walton's argumentation schemes and to solve some of their dissatisfactions.
\end{abstract}

Keywords: Reasons. Judgments. Arguments. Concept application. Cognition.

\title{
I. Conceptos y argumentación
}

Una de las capacidades cognitivas centrales de los seres humanos es la capacidad de formar juicios. Cuando formamos un juicio, asumimos cierto compromiso acerca de la realidad de un determinado hecho. Por ejemplo, podemos sin dificultad juzgar que el resultado de la adición de 3 a 5 es 8 . En este caso, el hecho acerca de cuya realidad juzgamos es un hecho matemático. Otros hechos son más difíciles de juzgar: piénsese, por ejemplo, en la existencia de componentes últimos e indivisibles de la materia. Como podemos ver con estos ejemplos, la posesión efectiva de la capacidad de formar un determinado juicio depende del contenido mismo del juicio, es decir de aquello de lo cual el juicio trata. Los conocimientos y competencias requeridos para poder juzgar que la suma de 3 y 5 es 8 no son evidentemente los mismos que los conocimientos y competencias requeridos para poder juzgar que existen componentes últimos e indivisibles de la materia. Cada uno de estos juicios pertenece a ámbitos distintos de actividad y conocimiento; pero, sobre todo, el contenido de cada uno de estos juicios está constituido de nociones distintas. Por un lado, tenemos las nociones de adición y de los números 3, 5 y 8 . Por el otro, tenemos las nociones de materia, 
componentes últimos e indivisibilidad. Las nociones que conforman el contenido de un juicio - o de otras actitudes proposicionales - son llamadas conceptos.

Todo concepto tiene un valor semántico, es decir aquello a lo que el concepto refiere o que es representado por el concepto. Por ejemplo, el valor semántico de un concepto general como el concepto manzana es la propiedad de ser una manzana. Es también posible identificar el valor semántico de un concepto general con su extensión, esto es admitir que el valor semántico del concepto manzana - nuestro ejemplo - es la totalidad de las manzanas existentes. Sin embargo, esta manera de concebir el valor semántico de este tipo de conceptos conlleva ciertas dificultades, pues es razonable sostener - para seguir con nuestro ejemplo - que la extinción de las manzanas no implica la extinción del valor semántico del concepto manzana. En otras palabras, la extinción de las manzanas no nos impide formar juicios perfectamente inteligibles acerca de las manzanas. Tal vez está dificultad podría ser superada si se admitiera que la extensión del concepto manzana es el conjunto de todas las manzanas existentes, las que han existido y las que existirán en el futuro. Pero si nos preguntamos en qué se fundamenta la unidad de semejante conjunto, debemos admitir que sus miembros - sean pasados, presentes o futuros - son objetos que comparten más o menos las mismas propiedades, pero, sobre todo, la propiedad de ser una manzana. Dado el papel fundamental de la propiedad de ser una manzana en la determinación misma de la extensión del concepto manzana, parece correcto sostener que el valor semántico de este concepto es, precisamente, la propiedad de ser una manzana.

Además de conceptos generales, existen conceptos singulares, es decir conceptos que refieren a individuos en particular, como por ejemplo: el barco de Teseo, Angela Merkel, el número 2 o Azerbaiyán. Evidentemente, cada uno de estos conceptos refiere a individuos con distintas condiciones de identidad, pero todos ellos son individuos auténticos, cada uno a su manera. Por último, podemos añadir a nuestra lista de tipos de conceptos los conceptos que representan operaciones lógicas como la negación, la conjunción, la cuantificación, etcétera. Cada uno de estos conceptos lógicos suele ser expresado por diversos términos de la lengua. Desde luego, otros tipos de conceptos pueden tal vez ser incluidos en la lista. Pero más allá de los tipos de conceptos que sea 
posible distinguir, y del valor semántico que se les pueda asignar, el punto que queremos resaltar es el siguiente: todo concepto, sea general, singular o lógico, además de valor semántico, posee determinadas condiciones de aplicación.

Aplicar un concepto no es otra cosa que formar un juicio en cuyo contenido figura dicho concepto (Peacocke, 1992), además de otros conceptos que también han sido aplicados. Por ejemplo, para formar un juicio perceptivo cuyo contenido es esta manzana no está madura, es necesario aplicar cuatro conceptos: un concepto demostrativo singular basado en una experiencia perceptiva, los conceptos generales manzana y estar madura y el concepto lógico negación. La aplicación de cada uno de estos conceptos no se produce de manera arbitraria, sino que refleja la posesión de capacidades que obedecen a restricciones o normas precisas. Si una persona desea seleccionar, entre las diversas frutas que hay en el interior de una canasta, una manzana madura, debe poder distinguir, en primer lugar, cada uno de los objetos individuales que se encuentran en la canasta. La persona debe también poder distinguir al menos algunas de las propiedades perceptibles de dichos objetos, como la forma, la textura, el color, el aroma, etcétera. Ser capaz de distinguir perceptivamente individuos y sus propiedades es una condición necesaria para la aplicación de conceptos demostrativos singulares basados en experiencias perceptivas. Además de lo anterior, la persona debe poder saber cuándo un objeto es una manzana y cuándo una fruta de este tipo, a diferencia de frutas de otro tipo, está madura. Finalmente, dado que es plausible sostener que una persona no es capaz de saber cuándo una manzana está madura sin ser al mismo tiempo capaz de saber cuándo una manzana no está madura (ni es tampoco capaz de saber cuándo un objeto es una manzana sin ser al mismo tiempo capaz de saber cuándo un objeto no es una manzana), podemos afirmar que las mismas capacidades que permiten a la persona saber cuándo una manzana está madura le permiten también saber - o al menos le aportan los elementos necesarios para saber - cuándo aplicar la negación al juicio esta manzana está madura (Peacocke, 2008). Así pues, solamente en la medida en que la persona posee todas estas capacidades, cada una con sus restricciones o normas específicas, puede seleccionar una manzana de la canasta (por la 
razón de que ha logrado identificarla como una manzana) y juzgar que la manzana en cuestión no está madura, es decir formar un juicio cuyo contenido es esta manzana no está madura.

El conjunto de restricciones o normas que regulan la aplicación de un determinado concepto es aquello en lo que consisten las condiciones de aplicación de dicho concepto.

La capacidad de aplicar conceptos es mucho más fundamental que la capacidad de clasificar objetos. Cuando clasificamos objetos, podemos aplicar (o intentar aplicar) cualquier tipo de criterio, por ejemplo: ser bípedo, ser de color rojo, ser de color rojo con puntos azules, ser alguno de los perros que escuché ladrar el 02 de febrero de 1997, ser un objeto inclasificable, etcétera. La aplicación de criterios de clasificación supone siempre la aplicación previa de conceptos. Así, la aplicación de los ejemplos anteriores de criterios de clasificación supone la aplicación previa, entre otros, de los conceptos bípedo, rojo, azul, perro, ladrar, 02 de febrero de 1997 e inclasificable. La aplicación de un concepto, en cambio, no supone necesariamente la aplicación previa de otros conceptos, pues de otra manera nos veríamos conducidos a una regresión ad infinitum de aplicación de conceptos. La aplicación de un concepto puede, en algunos casos, suponer la aplicación previa de otros conceptos, pero no en todos los casos. Pero, sobre todo, el propósito de la aplicación de conceptos no es la agrupación de objetos que satisfagan un determinado criterio, sino la formación de juicios. Como ya señalamos, formar un juicio es asumir cierto compromiso acerca de la realidad de un determinado hecho; ser capaz de asumir tal forma de compromiso es una condición fundamental de la que depende nuestra capacidad de clasificar objetos. Las capacidades de aplicar conceptos y de clasificar objetos no pueden, por consiguiente, ser identificadas entre sí. ${ }^{1}$

Dado que aplicar un concepto no es otra cosa que formar un juicio en cuyo contenido figura dicho concepto, las condiciones que regulan la aplicación de conceptos deben igualmente regular los procesos de formación de juicios. En efecto, para ser realmente capaz de formar un determinado juicio en particular, es necesario ser sensible a las restricciones o normas que derivan

\footnotetext{
${ }^{1}$ Para una fundamentación más detallada de la distinción entre aplicación de conceptos y clasificación de objetos, véase Millikan (2000), en particular el capítulo 3.
} 
de - o que están vinculadas a - los conceptos que constituyen el contenido del juicio en cuestión. De ahí que la capacidad de juzgar que la suma de 3 y 5 es 8 sea distinta de la capacidad de juzgar que existen componentes últimos e indivisibles de la materia, pues los distintos conceptos que constituyen los contenidos respectivos de estos juicios imponen condiciones de aplicación que obedecen a distintas normas o restricciones.

Pero, ¿qué tienen que ver todas estas reflexiones acerca de los conceptos y sus mecanismos de aplicación con el tema principal de nuestro trabajo, a saber, la argumentación? La tesis central sobre la que se apoya el presente trabajo es que la sensibilidad a las condiciones de aplicación de nuestros conceptos - sensibilidad que adquirimos con el desarrollo de nuestras capacidades conceptuales - es el factor principal que explica nuestra capacidad de producir y evaluar razones cuando argumentamos. Esta tesis es respaldada por las siguientes premisas, algunas de las cuales ya han sido mencionadas:

1) Cuando argumentamos, expresamos razones con el propósito de inducir en nuestro interlocutor la formación o la revisión de un determinado juicio, o con el propósito de resistir a la revisión de un juicio ante las críticas de nuestro interlocutor.

2) Formar juicios es aplicar conceptos.

3) Todo concepto posee condiciones de aplicación.

4) Poseer un concepto es ser capaz de aplicarlo, es decir ser sensible a sus condiciones de aplicación.

De acuerdo con la premisa (1), cuando expresamos razones en un intercambio argumentativo, podemos tener alguno de los siguientes propósitos:

a) Inducir en nuestro interlocutor la formación de un determinado juicio.

b) Inducir en nuestro interlocutor la revisión de un determinado juicio.

c) Resistir a la revisión de un juicio ante las críticas de nuestro interlocutor. 
Dada la identidad, que sostenemos en la premisa (2), entre formación de juicios y aplicación de conceptos, podemos reformular los propósitos de la enunciación de razones en un intercambio argumentativo de la siguiente manera:

a') Inducir en nuestro interlocutor la aplicación de un determinado concepto.

b') Inducir en nuestro interlocutor la renuncia a aplicar un determinado concepto.

c') Resistir a la renuncia a aplicar un determinado concepto ante las críticas de nuestro interlocutor.

Como es señalado en la premisa (3), todo concepto posee condiciones de aplicación, es decir a todo concepto están asociadas restricciones o normas que determinan cuándo el concepto puede o debe ser aplicado y, por consiguiente, cuándo el concepto no puede o no debe ser aplicado. De esta afirmación podemos concluir que, tanto para inducir en nuestro interlocutor la aplicación de un determinado concepto o la renuncia a su aplicación, como para evitar tener que renunciar nosotros mismos, ante las críticas de nuestro interlocutor, a la aplicación de un determinado concepto, es necesario regular nuestros procesos de producción y evaluación de razones de acuerdo con las condiciones de aplicación de los conceptos correspondientes en cada caso. Según la premisa (4), en la medida en que poseemos los conceptos que constituyen el contenido del juicio cuya formación o revisión queremos inducir en nuestro interlocutor, o a cuya revisión queremos resistir, somos sensibles a las condiciones de aplicación de dichos conceptos, es decir somos capaces de reconocer cuándo los conceptos deben ser aplicados y, también, cuándo no deben ser aplicados. Así pues, la posesión de estos conceptos implica la posesión de las capacidades necesarias tanto para identificar las razones que permiten efectivamente inducir en nuestro interlocutor la formación o la revisión del juicio en cuestión, como para evaluar las razones que formula nuestro interlocutor con el propósito de inducir en nosotros mismos la revisión de nuestro propio juicio. ${ }^{2}$

\footnotetext{
2 Para una elaboración más detallada de estos puntos, que se apoya principalmente en la teoría de los conceptos de Peacocke $(1992,2008)$, véase (texto del autor).
} 
En resumen, nuestra postura es que la posesión de conceptos es el factor principal que explica nuestra capacidad de producir y evaluar razones cuando argumentamos o, para decirlo de otro modo, nuestro dominio de la dimensión lógica de la argumentación. Además de una dimensión lógica, podemos distinguir, en el fenómeno social de la argumentación, una dimensión dialéctica y una dimensión retórica. La dimensión dialéctica se refleja en la necesidad, cuando argumentamos, no simplemente de producir razones, sino de responder racionalmente a un interlocutor que manifiesta críticamente su desacuerdo y que posee una perspectiva y objetivos propios. La dimensión retórica, en cambio, se refleja en la necesidad de formular nuestras razones de manera tal que su relevancia en la discusión sea claramente expuesta y que puedan lograr, como consecuencia de ello, su objetivo de persuasión racional (Wenzel, 2006). Desde nuestro enfoque, la dimensión lógica de la argumentación, en cuanto a ella, se refleja en la necesidad, cuando argumentamos, de identificar razones que, dado el contexto social y cultural de la discusión, la perspectiva de nuestro interlocutor y la manera en que dichas razones son expresadas, sean capaces de inducir en nuestro interlocutor la formación o la revisión de un determinado juicio, o nos permitan resistir, ante las críticas de nuestro interlocutor, a la revisión de un juicio que nosotros mismos hemos formado. Para satisfacer estos propósitos, como hemos visto, es necesario que nuestros procesos de producción y evaluación de razones sean regulados por las condiciones de aplicación de los conceptos que constituyen el contenido de los juicios relevantes en el intercambio argumentativo en el que participamos. Tal regulación es posible en virtud, fundamentalmente, de la posesión de estos mismos conceptos.

Una vez dicho lo anterior, el objetivo principal de nuestro trabajo puede ser claramente enunciado: se trata de comparar nuestra propuesta acerca de la manera en que poseemos dominio de la dimensión lógica de la argumentación con una de las propuestas más influyentes en la actualidad, en el campo de la teoría de la argumentación, acerca de esta misma cuestión, esto es la propuesta de los esquemas de argumentación, principalmente defendida por Douglas Walton (Walton, 1996, 2013; Walton, Reed \& Macagno, 2008). Según Walton, cuando argumentamos, seguimos ciertos patrones de inferencia que pueden ser formulados y clasificados en esquemas 
generales de argumentación, cada uno con sus características propias. En una buena parte de su obra, Walton se da a la tarea de identificar y describir dichos esquemas. El proyecto de identificación y descripción de esquemas de argumentación, tal como se lo plantea el propio Walton, no se limita, sin embargo, a la elaboración de un reporte de regularidades observadas en la manera en que las personas proceden cuando argumentan, independientemente de si lo hacen correctamente o no, sino que dicho proyecto tiene ambiciones normativas: se trata de identificar y describir esquemas correctos de argumentación, o esquemas que pueden ser correctos si son empleados adecuadamente. En otras palabras, dichos esquemas son, además de patrones comunes observados en los intercambios argumentativos, instrumentos de evaluación de la argumentación misma (Walton et al., 2008, p. 11). Desde la perspectiva de Walton, ser capaz de producir y evaluar razones en un intercambio argumentativo, es decir tener dominio de la dimensión lógica de la argumentación, es ser capaz de emplear adecuadamente esquemas de argumentación.

Pero, además de comparar nuestra propuesta con la propuesta de Walton, y distinguirla de ella, deseamos también mostrar sus ventajas, pues no solamente se trata, a nuestro parecer, de una propuesta más simple que fundamenta la capacidad de producir y evaluar razones en una capacidad cognitiva básica humana, esto es la capacidad de formar juicios, y que explica al mismo tiempo la absoluta generalidad de nuestra capacidad de argumentar, es decir el hecho de que podamos argumentar sobre prácticamente cualquier tema (en la medida en que poseemos sus conceptos), sino que nuestra propuesta permite igualmente solucionar y/o explicar algunas de las insatisfacciones que surgen en la formulación de esquemas de argumentación. Sin embargo, antes de pasar a los detalles de esta confrontación de planteamientos, queremos brevemente aclarar algunos puntos acerca de las premisas que sustentan nuestra tesis central.

\section{Algunas clarificaciones}

En nuestro primer comentario aclaratorio, queremos señalar que, además de permitirnos inducir la formación o la revisión de juicios en nuestro interlocutor y resistir a la revisión de nuestro propios juicios, la argumentación nos ayuda a realizar muchos otros propósitos más: lograr acuerdos en una 
negociación, generar una venta, justificar nuestras acciones y conservar una buena reputación, coordinar las acciones de un grupo, resolver disputas en todo tipo de disciplinas teóricas, resolver problemas familiares y laborales, etcétera. Tales propósitos tienen, desde luego, una gran importancia en nuestra vida personal, social y profesional. Sin embargo, es preciso resaltar que, cuando se emplea la argumentación, el éxito en la realización de estos propósitos depende fundamentalmente del éxito en la realización de los propósitos de inducir la formación o la revisión de juicios en nuestro interlocutor y/o de resistir a la revisión de nuestros propios juicios ante las críticas que nuestro interlocutor formula. Por tal motivo, estos últimos propósitos pueden ser considerados los objetivos propios de la argumentación, aun si, en el curso de un intercambio argumentativo, pueden ser abandonados en todo momento para favorecer otro tipo de métodos no argumentativos, y aun si las personas mismas que argumentan no se los representan como sus propósitos primarios.

Los comentarios aclaratorios siguientes nos conducen a cuestiones relativas, no a la argumentación, sino a la naturaleza de los conceptos. No obstante, es importante formularlos para mostrar, por un lado, que nuestra propuesta no está vinculada a ninguna visión particular de los conceptos $y$, por el otro, que su adopción nos permite conectar la teoría de la argumentación con distintas cuestiones que se discuten en filosofía de la mente acerca del pensamiento, el lenguaje y la racionalidad. El primer punto que queremos resaltar es que el hecho de admitir que formar juicios es aplicar conceptos no nos obliga a aceptar que la posesión de conceptos - vistos como componentes de contenidos posibles de juicios - deba de algún modo anteceder a la posesión misma de la capacidad de formar juicios, ni tampoco que la capacidad de formar juicios deba anteceder a la posesión de conceptos. Un partidario de una propuesta como la de Robert Brandom (1994, 2000), por ejemplo, puede aceptar que formar juicios es aplicar conceptos, al mismo tiempo que sostiene que la intencionalidad primaria debe situarse, no en el pensamiento, sino en el lenguaje y que la identificación de componentes conceptuales deriva completamente del análisis de actos primarios de enunciación de aserciones conectadas inferencialmente entre sí. En una visión teórica de este tipo, la adquisición de conceptos derivaría de la adquisición del lenguaje o, dicho de 
otro modo, es de la adquisición socialmente regulada de la capacidad de producir aserciones que derivaría nuestra compresión implícita de las conexiones inferenciales que existen entre dichas aserciones y, a través de tal comprensión, derivaría también nuestra captación de las restricciones y normas que regulan el uso de los componentes conceptuales que constituyen el contenido de las aserciones que producimos. Así pues, aunque nuestra propuesta fue inicialmente inspirada por el trabajo de Christopher Peacocke $(1992,1999,2004)$, su adopción no nos obliga a aceptar la visión de los conceptos que defiende este autor, en particular la idea según la cual los conceptos poseen una naturaleza propia, relativamente independiente del lenguaje, que es posible captar a través de la formulación de condiciones necesarias y suficientes para su individuación, o al menos para la individuación de algunos de ellos, sea en términos de condiciones de justificación, es decir en virtud de la identificación de las razones básicas que autorizan su aplicación (Peacocke, 1992), o en términos de condiciones de determinación de su valor semántico, es decir en virtud de la identificación de su modo particular de contribución a las condiciones de verdad de los contenidos proposicionales en los que figuran (Peacocke, 2008). Estas consideraciones muestran también que el hecho de admitir que formar juicios es aplicar conceptos no nos obliga a aceptar ni la primacía del pensamiento sobre el lenguaje, ni la del lenguaje sobre el pensamiento.

El segundo punto que deseamos abordar tiene que ver con la idea de que todo concepto posee condiciones de aplicación: admitir tal cosa no nos obliga a aceptar alguna concepción particular del origen y la naturaleza de las restricciones o normas en las que consisten dichas condiciones. De nuevo, si preferimos adoptar una propuesta como la de Robert Brandom, podemos sostener que las restricciones o normas que regulan la aplicación de conceptos derivan de las restricciones o normas socialmente impuestas que regulan la enunciación de aserciones. Además de tales restricciones o normas socialmente impuestas, las restricciones o normas que regulan la aplicación de conceptos podrían también haber derivado, al menos en parte, de las restricciones o normas que regulan nuestra actividad cognitiva, las cuales se han establecido a lo largo del desarrollo evolutivo de nuestra especie (Mercier \& Sperber, 2017). Estas dos opciones son compatibles con la idea de Quine (1954) según la cual la impresión que podemos tener de la 
analiticidad de enunciados como "todo lo que tiene color es extenso" o "los solteros son hombres no casados", es decir la impresión de que estos enunciados reflejan restricciones o normas que regulan el uso de ciertos términos (o la aplicación de ciertos conceptos), derivaría de meros factores contingentes (sociales, culturales y/o psicológicos), y no del puro significado de los términos que componen dichos enunciados. Nuestra propuesta puede también ser adoptada, sin embargo, por aquellos teóricos que deseen sostener que la analiticidad de un enunciado, o de un juicio, deriva de la identidad misma de los conceptos que constituyen su contenido. En este caso, las restricciones o normas que regulan la aplicación de nuestros conceptos derivarían también, como en el caso de la teoría de Peacocke, de la identidad de dichos conceptos. El punto que queremos aquí resaltar es que nuestra propuesta no favorece más una perspectiva que otra.

Nuestro tercer punto está relacionado con la polémica entre realismo y antirrealismo. Para Peacocke, todo juicio tiene como propósito esencial la verdad (Peacocke, 1992, 1999, 2004, 2008), es decir que "es un propósito constitutivo del acto de juzgar que intentamos juzgar que $p$ sólo si es verdadero que $p^{\prime \prime}$ (Peacocke, 2004, p. 13; mi traducción). Dado que formar juicios es aplicar conceptos, si adoptamos el punto de vista de Peacocke, debemos sostener que la verdad es igualmente el propósito esencial de la aplicación de conceptos. Por consiguiente, las restricciones o normas que regulan la aplicación de conceptos deben necesariamente operar de forma tal que contribuyan a la formación de juicios verdaderos. En congruencia con estas ideas, Peacocke defiende una concepción realista del lenguaje y el pensamiento. Sin embargo, el hecho de admitir que formar juicios es aplicar conceptos no nos obliga a adoptar una visión realista de este tipo, pues admitir que formar jucios es aplicar conceptos no nos obliga a aceptar que el propósito esencial de la formación de juicios es la verdad. Nuestra propuesta es ciertamente compatible con la adopción de una concepción realista del lenguaje y el pensamiento, pero también con una concepción antirrealista, en la medida en que deja abierta la posibilidad de rechazar la idea de que la verdad es el propósito esencial de la formación de juicios y de sostener que la formación de juicios obedece a algún otro propósito fundamental. 
Nuestro cuarto y último punto tiene que ver con la última de nuestras premisas: la idea de que poseer un concepto es ser capaz de aplicarlo, es decir la idea de que una persona no puede poseer un concepto sin ser al mismo tiempo sensible a sus condiciones de aplicación y, por consiguiente, sin ser al mismo tiempo capaz de formar adecuadamente juicios en cuyo contenido figura dicho concepto.

Algunos autores, como Jerry Fodor (1998), sostienen que un concepto es, básicamente, una representación formada en nuestra mente o en nuestro cerebro. Así, de acuerdo con Fodor, poseemos el concepto gato, por ejemplo, cuando pueden ser formadas, en nuestra mente o en nuestro cerebro, representaciones de la propiedad de ser un gato, es decir estructuras singulares, mentales o cerebrales, que representan dicha propiedad. Para que tales estructuras puedan representar la propiedad de ser un gato, según la teoría de Fodor (1990), basta con que dos condiciones sean satisfechas: (i) que se trate de estructuras de un tipo T, la formación (o estimulación) de cuyas instancias, o tokens, covaríe nomológicamente con la estimulación de nuestros organos sensoriales causada por la presencia en nuestro entorno de entidades que poseen la propiedad de ser un gato, y (ii) que, de la existencia de estructuras de tipo T, dependa la existencia de estructuras de cualquier otro tipo cuya formación (o estimulación) resulte también de la estimulación de nuestros organos sensoriales causada por la presencia en nuestro entorno de entidades que poseen la propiedad de ser un gato (esto es: la famosa cláusula fodoriana de dependencia causal asimétrica). Hay, en la teoría de Fodor, puntos complejos y polémicos que, ciertamente, no podemos abordar aquí. No obstante, la idea sobre la que deseamos enfocarnos es más bien sencilla: de acuerdo con la propuesta de Fodor, poseer un concepto no implica necesariamente ser sensible a sus condiciones de aplicación, ni ser capaz de formar adecuadamente juicios en cuyo contenido figura dicho concepto, sino simplemente poseer una mente o un cerebro que, en virtud de ciertas relaciones nomológicas con su entorno, permita la formación de estructuras causalmente conectadas con aquello que estas mismas estructuras, en virtud de tal conexión, representan. 
En la premisa (4) que sirve de sustento a nuestra tesis central se afirma que poseer un concepto es ser capaz de aplicarlo. Así, de acuerdo con esta premisa, poseer un concepto es - o al menos implica - poseer cierto tipo de capacidad. En cambio, para Fodor, un concepto es una representación interna y una representación interna es un tipo de objeto (mental particular). Consecuentemente, según la perspectiva de Fodor, poseer un concepto es poseer cierto tipo de objeto (Fodor, 1998). Para Fodor, es fundamental que una representación sea un tipo de objeto pues, por un lado, según su propia teoría, una entidad es una representación en virtud de sus relaciones causales con aquello que representa $y$, por el otro, solamente un objeto puede tener relaciones causales. Si poseer un concepto es simplemente poseer cierto tipo de objeto, poseer un concepto no implica poseer capacidad alguna. Un organismo puede, por consiguiente, poseer un determinado concepto sin que dicho concepto le sea de utilidad, es decir sin que dicho concepto pueda tener alguna función para él o para alguno de sus subsistemas. Esta consecuencia de la teoría de Fodor nos parece, sin embargo, problemática: la función específica de un concepto debe estar ligada a su propia identidad, es decir a aquello que hace que el concepto en cuestión sea distinto de todos los demás y, por consiguiente, a aquello en lo que consiste poseer este concepto a diferencia de cualquier otro. Por otro lado, la función de un concepto no puede ser reducida a meras relaciones causales, pues nuestros conceptos no son simples objetos físicos, sino que tienen una significación en nuestra actividad cognitiva, la cual no es simplemente un conjunto de procesos físicos como el ciclo del agua o la fusión nuclear.

Todos estos problemas, obviamente, no pueden ser abordados en este trabajo. Limitémonos a señalar, pues, que la adopción de la teoría de Fodor no conlleva necesariamente el rechazo de nuestra propuesta. Si decidimos adoptar la teoría de Fodor, a pesar de sus serias dificultades, no podremos, desde luego, sostener la idea de que poseer un concepto es ser capaz de aplicarlo, pero podremos, en cambio, admitir que, si bien es posible poseer un concepto sin ser capaz de aplicarlo, no es posible adquirir la capacidad de aplicar un concepto sin adquirir la sensibilidad adecuada a sus condiciones de aplicación, es decir sin adquirir la capacidad de formar adecuadamente juicios en 
cuyo contenido figura dicho concepto. ${ }^{3}$ Desde nuestra enfoque, adquirir tal forma de sensibilidad es aquello en lo que consiste adquirir un concepto. Sin embargo, no es necesario, al menos en este momento, entrar en polémicas al respecto, pues nuestra propuesta, con la modificación señalada, puede ser aceptada incluso por los partidarios del enfoque de Fodor. ${ }^{4}$ Aunque es posible incorporar esta modificación en la formulación de nuestra propuesta, por motivos expositivos, no haremos empero mención de ella en el resto de este trabajo.

\section{Esquemas}

Pasemos ahora al análisis de los esquemas de argumentación. De acuerdo con Walton, los esquemas de argumentación son "las formas de argumento (estructuras de inferencia) que permiten identificar y evaluar tipos comunes de argumentación en el discurso cotidiano" (Walton et al., 2008, p. 11; mi traducción). Así pues, como ya fue mencionado, para Walton, los esquemas de argumentación tienen a la vez una función descriptiva y una función normativa: no solamente nos permiten identificar tipos comunes de argumentación, sino también evaluar los argumentos producidos. Veamos el ejemplo del esquema de argumentación que Walton (2013, p. 6; mi traducción) denomina "argumento por opinión de experto" (argument from expert opinion):

Premisa mayor: el informante $E$ es un experto en el campo de conocimiento $S$ el cual contiene la proposición $A$.

Premisa menor: $E$ afirma que la proposición $A$ es verdadera (falsa).

Conclusión: $A$ es verdadera (falsa).

Walton sostiene que un esquema como el que acabamos de presentar constituye un patrón común en la argumentación cotidiana; también sostiene que se trata de un esquema correcto de

\footnotetext{
${ }^{3}$ En términos que un partidario de las ideas de Fodor preferiría: no es posible tener la capacidad de poner en nuestra belief box una representación mental cuyo contenido es esta manzana no está madura, por ejemplo, sin ser al mismo tiempo sensible a las condiciones de aplicación del concepto manzana (y a las condiciones de aplicación del resto de los conceptos que constituyen el contenido proposicional de dicha representación mental).

${ }^{4}$ Para una crítica de la teoría de Fodor y de otras teorías similares, véase (texto del autor).
} 
argumentación el cual, sin embargo, como todo esquema de argumentación, puede ser cuestionado y refutado. Una conclusión que es respaldada por el uso de un determinado esquema de argumentación no es, pues, establecida de manera definitiva, sino que puede ser abandonada si, en un proceso dialéctico de intercambio de razones, el uso del esquema es refutado. Para mostrar cómo el uso de un esquema puede ser refutado, Walton asocia una serie de preguntas críticas a cada uno de los esquemas identificados. En el caso del "argumento por opinión de experto", la lista de preguntas críticas asociada es la siguiente (Walton, 2013, p. 6; mi traducción):

$\mathrm{PC}_{1}$ : Pregunta sobre la especialización. ¿Qué tan confiable es $E$ en tanto que experto?

PC $_{2}$ : Pregunta sobre el campo. ¿Es $E$ un experto en el campo de conocimiento al que $A$ pertenece?

$\mathrm{PC}_{3}$ : Pregunta sobre la opinión. ¿Qué afirmó $E$ que implica $A$ ?

$\mathrm{PC}_{4}$ : Pregunta sobre la confiabilidad. ¿Es $E$ fiable personalmente como informante?

PC $_{5}$ : Pregunta sobre la consistencia. ¿'Es A consistente con lo que otros expertos afirman?

$\mathbf{P C}_{6}$ : Pregunta sobre el respaldo en evidencia. ¿La afirmación de $E$ está basada en evidencia?

Una persona que desee refutar el uso, en una ocasión particular, del "argumento por opinión de experto" puede plantear alguna de las preguntas críticas mencionadas. Si la persona que formula el argumento no es capaz de dar una respuesta apropiada, la conclusión podrá ser rechazada. Sin embargo, si la pregunta es adecuadamente respondida, la conclusión podrá ser mantenida, a menos que otra pregunta crítica sea planteada. Así, según Walton, las preguntas críticas indican, para cada tipo específico de esquema de argumentación, el camino a seguir en un intercambio dialéctico entre dos o más personas que argumentan y que, en virtud de esta dinámica de cuestionamientos, sitúan y resitúan la carga de la prueba en su respectivo contrincante hasta llegar, al menos en algunos casos, a una resolución.

Dado que, para Walton, un esquema de argumentación no es simplemente una herramienta más de análisis y evaluación de argumentos, sino que constituye un patrón común de argumentación en el discurso cotidiano, es razonable suponer que, desde la perspectiva de Walton, 
nuestro dominio de la dimensión lógica de la argumentación puede ser explicado en virtud de nuestra capacidad de utilizar adecuadamente esquemas de argumentación. En efecto, el uso de esquemas de argumentación no es una mera recomendación de Walton para mejorar nuestras habilidades argumentativas, sino que, aparentemente, empleamos ya de algún modo dichos esquemas. El uso de esquemas de argumentación podría, pues, explicar nuestra capacidad de identificar razones para respaldar o refutar aserciones en un intercambio argumentativo. Es precisamente contra esta idea que deseamos dirigir nuestros argumentos, pues nosotros sostenemos que nuestra capacidad de identificar razones en un intercambio argumentativo se fundamenta, no en el uso de algún tipo de esquemas, sino en la simple posesión de conceptos.

Sin embargo, tal vez esta manera de caracterizar la propuesta de Walton impone propósitos ambiciosos a su teoría que el mismo Walton nunca ha deseado adoptar. Es posible que Walton no haya intentado en ningún momento explicar nuestro dominio de la dimensión lógica de la argumentación, sino que simplemente desea poner a nuestra disposición, en tanto que teóricos y docentes de la argumentación, un conjunto de herramientas que permiten a la vez describir ciertos aspectos de nuestras prácticas argumentativas, evaluar los argumentos que resultan de dichas prácticas y diseñar técnicas y estrategias para mejorarlas. La explicación de nuestro dominio de la dimensión lógica de la argumentación debe, pues, ser buscada en otro lado. Si esta interpretación de los propósitos de Walton es correcta, deseamos entonces sostener que nuestra propuesta proporciona precisamente dicha explicación: nuestra capacidad de identificar buenas razones para respaldar o refutar aserciones en un intercambio argumentativo es explicada en virtud de nuestra sensibilidad a las condiciones de aplicación de los conceptos que constituyen el contenido de nuestros juicios. Tal sensibilidad deriva de la mera posesión de dichos conceptos. A partir de la posesión de conceptos es posible explicar, de hecho, el uso de esquemas de argumentación.

Veamos de nuevo el "argumento por opinión de experto". En este ejemplo de esquema de argumentación, es más bien fácil constatar que la validez del esquema deriva totalmente del uso del concepto experto en la premisa mayor: 
Premisa mayor: el informante $E$ es un experto en el campo de conocimiento $S$ el cual contiene la proposición $A$.

En efecto, parece correcto sostener que una persona no puede poseer el concepto experto si no ha podido de algún modo captar que un experto es una autoridad en un campo específico de conocimiento. La autoridad que un experto tiene en su campo de conocimiento constituye una razón para aceptar prima facie una proposición que el experto afirma si dicha proposición pertenece al campo de conocimiento en cuestión. Así, una persona que admite que $E$ es un experto no comprende plenamente el tipo de propiedad que atribuye a $E$, es decir no sabe realmente en que consiste ser un experto, si no es capaz de captar que la atribución de dicha propiedad implica la atribución del tipo de autoridad señalada. La posesión del concepto experto parece necesariamente implicar, por consiguiente, la comprensión de la relación que existe entre la propiedad de ser un experto en un campo de conocimiento $S$ y la autoridad del experto dentro del campo $S$.

Ahora bien, supongamos que una persona $P$ que posee el concepto experto acepta que $E$ es un experto en el campo de conocimiento $S$ y sabe, además, que $A$ es una proposición que pertenece a S. En otras palabras, $P$ acepta la premisa mayor del "argumento por opinión de experto". Supongamos también que $P$ ha podido constatar que $E$ afirma que $A$ es verdadera (falsa). Como consecuencia de ello, $P$ acepta la premisa menor del "argumento por opinión de experto":

Premisa menor: $E$ afirma que la proposición $A$ es verdadera (falsa).

En virtud de la información específica de la que dispone sobre el informante $E$ y la proposición $A$, y sobre todo en virtud de su posesión del concepto experto, $P$ puede aceptar prima facie que:

Conclusión: A es verdadera (falsa).

La posibilidad de captar la racionalidad de esta inferencia depende crucialmente de la posesión del concepto experto y del hecho de que este concepto ha sido aplicado a $E$. En efecto, una persona que posee el concepto experto es básicamente una persona que ha adquirido una forma adecuada 
de sensibilidad a las restricciones o normas que regulan la aplicación de este concepto, es decir a las restricciones o normas que constituyen sus condiciones de aplicación. En virtud del desarrollo de esta forma de sensibilidad, una persona que posee el concepto experto no puede aceptar las premisas del "argumento por opinión de experto" sin sentirse racionalmente obligada a aceptar, al menos prima facie, su conclusión.

Además de explicar la capacidad que las personas generalmente poseen de captar la racionalidad de la transición inferencial que da fundamento al "argumento por opinión de experto", la posesión del concepto experto y su aplicación a $E$ permiten también explicar y justificar el funcionamiento de las preguntas críticas que acompañan este mismo esquema. La primera impresión que podemos tener cuando observamos la lista de preguntas críticas que Walton asocia al "argumento por opinión de experto" es que no parece haber un principio que les de orden o que haya guiado de algún modo su selección. Algunas de las preguntas que encontramos en la lista, como $\mathrm{PC}_{2}$ y $\mathrm{PC}_{3}$, se limitan a cuestionar las premisas del argumento:

$\mathbf{P C}_{2}$ : Pregunta sobre el campo. ¿Es $E$ un experto en el campo de conocimiento al que $A$ pertenece?

$\mathrm{PC}_{3}$ : Pregunta sobre la opinión. ¿Qué afirmó $E$ que implica $A$ ?

Así, efectivamente, mientras que $\mathrm{PC}_{2}$ no hace más que cuestionar la premisa mayor del "argumento por opinión de experto", es decir el hecho de que $E$ sea realmente un experto en el campo de conocimiento al que $A$ pertenece, $\mathrm{PC}_{3}$ no hace más que cuestionar su premisa menor, es decir el hecho de que $E$ haya realmente afirmado $A$. El planteamiento de estas preguntas pierde sentido, sin embargo, una vez que el argumento ha sido considerado prima facie admisible, pues se trata de preguntas que deben formularse en un momento previo para poder determinar, precisamente, si el argumento es prima facie admisible (un argumento es prima facie admisible únicamente si sus premisas son prima facie aceptables). Una vez que el argumento ha sido considerado prima facie admisible, otro tipo de preguntas pueden plantearse, como las preguntas $\mathrm{PC}_{1}, \mathrm{PC}_{4}, \mathrm{PC}_{5}$ y $\mathrm{PC}_{6}$ :

PC $_{1}$ : Pregunta sobre la especialización. ¿Qué tan confiable es $E$ en tanto que experto? 


\begin{abstract}
PC 4 : Pregunta sobre la confiabilidad. ¿Es E fiable personalmente como informante?
PC $_{5}$ : Pregunta sobre la consistencia. ¿Es A consistente con lo que otros expertos afirman?

PC $_{6}$ : Pregunta sobre el respaldo en evidencia. ¿La afirmación de $E$ está basada en evidencia?
\end{abstract}

Aunque la lista de preguntas críticas que presenta Walton es un tanto arbitraria (¿por qué estas preguntas?, ¿por qué no otras?, ¿son todas las preguntas relevantes?), es posible, pues, al menos distinguir entre las preguntas críticas que pueden plantearse para determinar si el uso del "argumento por opinión de experto", en una ocasión particular, es prima facie admisible y las preguntas críticas que, en cambio, pueden plantearse una vez que el uso del esquema ha sido considerado prima facie admisible. Dicho en nuestra terminología: es posible distinguir entre las preguntas críticas que cuestionan la aplicación misma del concepto experto a $E$, así como la información específica que está contenida en las premisas (que $S$ es el campo de conocimiento en el cual $E$ es un experto, que $A$ es una proposición que pertenece a $S$ y que $E$ afirma que $A$ ), y las preguntas críticas que pueden plantearse una vez que la aplicación del concepto experto a $E$ y la información contenida en las premisas han sido ambas aceptadas. Tanto la relevancia de este segundo grupo de preguntas como su funcionamiento pueden ser explicados en virtud del hecho de que las partes que argumentan han admitido la aplicación del concepto experto a $E$.

Comencemos por $\mathrm{PC}_{1}$, esto es la pregunta “¿qué tan confiable es $E$ en tanto que experto?” Dado que, como ya mencionamos, la aplicación del concepto experto a $E$ implica el reconocimiento de la autoridad de $E$ en un campo específico de conocimiento, $\mathrm{PC}_{1}$ no hace más que cuestionar el hecho de que esta autoridad, en el caso particular de $E$, sea suficiente para aceptar las proposiciones que $E$ afirma, aun si dichas proposiciones pertenecen a su campo. La autoridad en un campo de conocimiento es algo que se puede tener en mayor o menor grado y es por ello que es lícito preguntarse si el grado de autoridad de $E$ en su propio campo es suficiente. Sin embargo, es importante también notar que, una vez que ha sido admitida la aplicación del concepto experto a $E$, la autoridad de $E$ debe ser reconocida, por lo que el hecho de cuestionarla puede interpretarse como un cuestionamiento de la aplicación misma del concepto experto a $E$. De ahí la impresión que 
se puede tener, cuando se lee $\mathrm{PC}_{1}$, de que dicha pregunta es un tanto necia o redundante, pues parece insistir en el cuestionamiento de un punto que ya había sido acordado, al menos implícitamente, cuando el uso del "argumento por opinión de experto" fue considerado prima facie admisible.

$\mathrm{PC}_{4}$, esto es la pregunta “¿es $E$ fiable personalmente como informante?”, cuestiona el hecho de que $E$ esté verdaderamente respaldando sus afirmaciones en el conocimiento especializado que posee. Dado que la autoridad de un experto, en tanto que experto, no está fundamentada en otra cosa más que en su conocimiento especializado, si se tienen sospechas legítimas de que $E$ no está respaldando sus afirmaciones en dicho conocimiento, la autoridad de $E$ deja inmediatamente de ser una razón para aceptar tales afirmaciones.

La pregunta "¿es $A$ consistente con lo que otros expertos afirman?", es decir PC 5 , es un tanto más compleja. Como ya señalamos, la autoridad de $E$ en un campo específico de conocimiento autoridad que necesariamente reconocemos cuando aplicamos el concepto experto a $E$ - constituye una razón para aceptar prima facie una proposición que $E$ afirma si dicha proposición pertenece al campo de $E$. Desde luego, el simple hecho de admitir que $E$ es un experto en un determinado campo de conocimiento no nos impide admitir que alguna otra persona, $E^{\prime}$ por ejemplo, sea también un experto en el mismo campo. Dado que en todo campo de conocimiento hay polémicas y que, por consiguiente, dos personas pueden ser plenamente expertos en un mismo campo de conocimiento aun si están en desacuerdo sobre la verdad de una proposición que pertenece a dicho campo, puede claramente presentarse una situación en la que, por un lado, el experto $E$ afirma que la proposición $A$ que pertenece a su campo es verdadera, mientras que, por el otro, el también experto $E^{\prime}$ en el mismo campo afirma que $A$ es falsa (o que alguna otra proposición incompatible con la verdad de $A$ es verdadera). En semejante situación, es obvio que la autoridad de $E^{\prime}-$ reconocida, al igual que la autoridad de $E$, cuando fue admitida la aplicación del concepto experto a $E^{\prime}$ - anula la autoridad de $E$ y, como consecuencia de ello, el hecho de que $E$ haya afirmado que $A$ es verdadera, aun si $A$ pertenece al campo de $E$, deja de ser una razón para aceptar la verdad de $A$. 
Finalmente, respecto de $\mathrm{PC}_{6}$, esto es de la pregunta “¿la afirmación de $E$ está basada en evidencia?", podemos claramente ver que esta pregunta no es muy distinta de $\mathrm{PC}_{4}$, pues ambas preguntas no hacen más que cuestionar el hecho de que $E$ esté respaldando sus afirmaciones en su conocimiento especializado, es decir en la evidencia que ha sido producida en el propio campo de $E$ y que suponemos que $E$, en tanto que experto, debe conocer.

Como es fácil constatar en la discusión de estos últimos párrafos, las preguntas críticas que formula Walton, cuando no cuestionan directamente las premisas del "argumento por opinión de experto", no hacen más que concentrarse en factores que limitan o anulan la autoridad que necesariamente atribuimos a $E$ cuando admitimos que $E$ es un experto, es decir cuando aplicamos el concepto experto a $E$. La identificación de nuevos factores de este tipo puede, por consiguiente, permitirnos formular nuevas preguntas críticas, por ejemplo: ¿ha declarado $E$ bajo amenaza de muerte que la proposición $A$ es verdadera?, ¿sufre $E$ de amnesias frecuentes tras algún accidente?, ¿tiene $E$ la obligación de mantener su conocimiento en secreto?, etcétera. El número de preguntas críticas que podemos añadir a la lista de Walton depende de los límites de nuestra imaginación. No obstante, como acabamos de señalar, el principio que nos permite producir estas preguntas es más bien simple: la identificación de factores o situaciones que limitan o anulan el tipo de autoridad que necesariamente atribuimos a $E$ cuando aplicamos el concepto experto a $E$. La pertinencia de este principio no es más que una consecuencia del hecho de que la racionalidad del "argumento por opinión de experto" depende esencialmente de la atribución de este tipo de autoridad a $E$.

Pero más allá del origen de las preguntas críticas y de la explicación de su funcionamiento, el punto que queremos resaltar es el siguiente: es fundamentalmente de la posesión del concepto experto que deriva tanto nuestra capacidad de captar la racionalidad del "argumento por opinión de experto" como nuestra capacidad de identificar preguntas críticas que nos permiten refutar el uso, en una ocasión particular, de este esquema de argumentación. En efecto, sostenemos que una persona que posee el concepto experto, en virtud simplemente de tal posesión, es capaz de:

a) aplicar el concepto a $E y$, por consiguiente, formar un juicio cuyo contenido es $E$ es un experto, 
b) captar que la aplicación del concepto experto a $E$ implica necesariamente el reconocimiento de la autoridad de $E$ en un campo específico de conocimiento,

c) captar que la autoridad de $E$, en tanto que experto, constituye una razón para aceptar prima facie una proposición que $E$ afirma si dicha proposición pertenece al campo de conocimiento de $E$,

d) $y$, finalmente, captar que el cuestionamiento de la autoridad de $E$, en tanto que experto, debilita o anula el derecho y/o la obligación racional de aceptar las proposiciones que $E$ afirma y que pertenecen a su campo.

Evidentemente, el hecho de admitir que la posesión del concepto experto implica la posesión de todas estas capacidades no nos obliga a admitir que una persona que posee el concepto experto debe tener una comprensión explícita del tipo de capacidades que posee en virtud de la posesión de este concepto. Las capacidades que una persona necesariamente posee en virtud de la posesión del concepto experto son capacidades que se reflejan fundamentalmente en la manera en que la persona está dispuesta a razonar cuando se cuestiona sobre la aplicación del concepto experto a un determinado individuo y, también, una vez que ha procedido a dicha aplicación. La idea que sostenemos es que una persona que carece de las capacidades (a), (b), (c) y (d) no puede ser una persona que posee el concepto experto y, por consiguiente, que la posesión del concepto experto implica la posesión de estas capacidades. De ahí que afirmemos que el uso, funcionamiento y racionalidad tanto del "argumento por opinión de experto" como de sus preguntas críticas estén fundamentados en la posesión del concepto experto.

Esta conclusión, desde luego, no se limita al caso particular del "argumento por opinión de experto", sino que se trata de una conclusión de alcance general: para todo esquema de argumentación $G$, existe al menos un concepto $C$ cuyas condiciones de aplicación explican la racionalidad tanto de $G$ como de las preguntas críticas que lo acompañan y cuya posesión explica nuestra capacidad de captar dicha racionalidad y nuestra capacidad de formular nuevas preguntas críticas. Así, sostenemos que el tipo de análisis que hemos efectuado en esta sección del 


\begin{abstract}
"argumento por opinión de experto" permite elucidar el funcionamiento y la racionalidad de cualquier otro de los esquemas de argumentación identificados por Walton. Veamos, por ejemplo, el "argumento por analogía” (Walton, 2013, p. 126; mi traducción):
\end{abstract}

Premisa de similaridad: en general, el caso $C_{1}$ es similar al caso $C_{2}$.

Premisa base: la proposición $A$ es verdadera (falsa) en el caso $C_{1}$.

Conclusión: la proposición $A$ es verdadera (falsa) en el caso $C_{2}$.

Este esquema de argumentación está acompañado de la siguiente lista de preguntas críticas (Walton, 2013, p. 142; mi traducción):

$\mathbf{P C}_{1}$ : ¿Hay diferencias entre $C_{1}$ y $C_{2}$ que tienden a anular la fuerza de su similitud?

$\mathrm{PC}_{2}$ : ¿¿s la proposición $A$ verdadera (falsa) en $C_{1}$ ?

$\mathrm{PC}_{3}$ : ¿ ¿Hay algún otro caso $C_{3}$ que es también similar al caso $C_{1}$, pero en el cual $A$ es falsa (verdadera)?

En el caso del "argumento por analogía", el concepto principal cuyas condiciones de aplicación explican tanto la racionalidad del esquema como la racionalidad de sus preguntas críticas es el concepto similar. La aplicación del concepto similar a $C_{1}$ y $C_{2}$, es decir la formación del juicio $C_{1}$ es similar a $C_{2}$, implica necesariamente la identificación - más o menos precisa y explícita - de un conjunto de propiedades que $C_{1}$ y $C_{2}$ comparten. Una persona que no es sensible a esta implicación de la aplicación del concepto similar a $C_{1}$ y $C_{2}$ no puede ser una persona que posea este concepto. De la existencia de esta implicación se sigue que no es posible proceder a la aplicación del concepto similar a $C_{1}$ y $C_{2}$ sin poseer y aplicar los conceptos de al menos algunas de las propiedades que comparten - o que se asume que comparten $-C_{1}$ y $C_{2}$. Por consiguiente, un análisis completo del "argumento por analogía" debe considerar, no solamente las condiciones de aplicación del concepto similar, sino también las condiciones de aplicación de los conceptos implicados en la aplicación misma del concepto similar, pues de estas últimas condiciones de aplicación derivan igualmente restricciones racionales que regulan el uso de dicho esquema. Sin embargo, más allá de 
estas complicaciones, es fácil ver que el hecho de que $C_{1}$ y $C_{2}$ compartan ciertas propiedades es una razón para aceptar prima facie que, si la proposición $A$ es verdadera (falsa) en $C_{1}$, la proposición $A$ es verdadera (falsa) en $C_{2}$.

Obviamente, por razones de espacio, no podemos aquí llevar a cabo el análisis completo del "argumento por analogía", ni mucho menos el análisis - parcial o completo - del resto de los esquemas de argumentación que discute Walton. Finalizaremos, pues, nuestro trabajo con una formulación breve de algunas críticas que se plantean, desde nuestra perspectiva, a la propuesta de Walton, críticas que esperamos desarrollar más detalladamente en otra ocasión.

\section{Observaciones críticas a la propuesta de Walton}

En la sección anterior, señalamos que la propuesta de Walton puede ser interpretada de dos maneras distintas: o bien suponemos que el uso de esquemas explica nuestro dominio de la dimensión lógica de la argumentación, es decir nuestra capacidad de identificar razones para respaldar nuestras aserciones o para refutar las aserciones de un interlocutor con el que estamos en desacuerdo, o bien suponemos que los esquemas de argumentación no tienen (ni se ha pretendido que tengan) tal poder explicativo, sino que simplemente constituyen herramientas útiles de análisis y evaluación de argumentos. La primera interpretación es, desde luego, más fácil de cuestionar que la primera, pues si se asume que el uso de esquemas es el factor principal que explica nuestro dominio de la dimensión lógica de la argumentación, es lícito entonces preguntar qué explica a su vez nuestra capacidad de emplear esquemas. El uso de esquemas de argumentación no parece ser una capacidad cognitiva básica de los seres humanos, o al menos no tan básica como la percepción o la aplicación de conceptos. Por otro lado, como hemos querido mostrar, nuestra capacidad de aplicar conceptos - una capacidad cognitiva básica humana - puede explicar tanto nuestra capacidad de captar la racionalidad de los esquemas de argumentación que propone Walton como nuestra capacidad de captar la racionalidad de las preguntas críticas asociadas a cada uno de estos esquemas. Nuestra capacidad de aplicar conceptos puede incluso explicar nuestra capacidad de identificar nuevas preguntas críticas, distintas de las preguntas críticas formuladas por Walton. Por 
lo tanto, es razonable admitir que lo que explica nuestro dominio de la dimension lógica de la argumentación no es el uso de esquemas, sino simplemente nuestra capacidad de aplicar conceptos.

Sin embargo, podría objetarse a este razonamiento que, mientras no se haya demostrado plenamente que la aplicación de conceptos explica la racionalidad de todo esquema de argumentación posible, así como nuestra capacidad de captar tal racionalidad, no es correcto asumir que la aplicación de conceptos explica - o es lo único que explica - nuestro dominio de la dimensión lógica de la argumentación. En efecto, en una de sus varias publicaciones, Walton (1996) presenta una lista de 29 esquemas de argumentación que incluye, además del "argumento por opinión de experto" y el "argumento por analogía", varios tipos de ad hominem, el "argumento por clasificación verbal”, el "argumento por precedente", los "argumentos por composición y división", el "argumento por opinión popular", etcétera. En publicaciones posteriores, Walton identifica varios esquemas de argumentación más, hasta llegar a una lista de 96 esquemas (Walton et al., 2008). ¿No es incorrecto suponer sin más que la racionalidad de cada uno de estos 96 esquemas es explicable en virtud de la simple aplicación de conceptos? ¿Y qué decir de los esquemas de argumentación que puedan ser identificados en el futuro? ¿Cómo garantizar que la aplicación de conceptos permite explicar la racionalidad de todos ellos?

Walton reconoce que, en lo que respecta a la identificación, formulación y clasificación de esquemas de argumentación, no ha procedido de forma sistemática, probablemente porque, según su propia opinión, se ha concentrado sobre todo en "preocupaciones prácticas en el manejo de casos reales" (Walton et al., 2008, p. 13; mi traducción). Los esquemas de argumentación que Walton ha podido identificar son aquellos que le han permitido analizar argumentos encontrados principalmente en textos periodísticos, o argumentos utilizados como ejemplos en manuales de lógica informal (Walton, 2013, p. 96). Desde nuestra perspectiva, esta falta de sistematicidad resulta, más que del hecho de que los intereses que han guíado la recolección de ejemplos han sido intereses prácticos, del hecho de que la identificación de esquemas de argumentación no es esencialmente otra cosa que la identificación - e intento de explicitación - de la manera en que las 
condiciones de aplicación de nuestros conceptos regulan racionalmente los procesos inferenciales que efectuamos. Los esquemas de argumentación que, al menos en principio, un teórico puede identificar y formular son tantos como los conceptos que poseemos y cuya aplicación puede ser relevante en distintas y variadas ocasiones, para algunos de estos conceptos, o solamente cuando se abordan cuestiones muy especializadas, para otros. De esto se sigue que los límites de la esfera de interacción social cuyas prácticas argumentativas deseamos estudiar restringen los esquemas de argumentación que podemos identificar, pues todo esfera de interacción social en la que se argumenta tiene sus propios conceptos relevantes y es fundamentalmente de estos conceptos que es posible derivar los esquemas de argumentación que supuestamente son empleados en la esfera estudiada. Los esquemas de argumentación que Walton ha podido identificar son, pues, los que derivan de los conceptos generalmente empleados en las prácticas argumentativas llevadas a cabo en la esfera de interacción social por la que dicho autor se ha principalmente interesado (textos periodísticos y manuales de lógica informal). A esto hay que añadir que, como es fácil constatar echando un vistazo a las propias listas de esquemas que Walton elabora, Walton da cierta prioridad en su análisis a la identificación de formas argumentales tradicionalmente estudiadas (argumentos ad hominem, ad populum, de autoridad, etcétera). Desde nuestra perspectiva, son más estos sesgos - conscientes o inconscientes - que explican la falta de sistematicidad de la propuesta de Walton que su interés por problemáticas de orden práctico en la argumentación.

Un factor adicional que explica la falta de sistematicidad de la propuesta de Walton tiene que ver con la dificultad que podemos encontrar cuando intentamos explicitar las condiciones de aplicación de nuestros conceptos. La posesión de un concepto implica necesariamente la sensibilidad a sus condiciones de aplicación, pero no necesariamente la capacidad de formular explícitamente tales condiciones. La sensibilidad a las condiciones de aplicación de un concepto se refleja principalmente, como ya mencionamos, en nuestras disposiciones a razonar de tal o cual manera en particular cuando consideramos la posible aplicación del concepto, o una vez que lo hemos aplicado, y no en nuestra capacidad de hacer explícitas las restricciones racionales que la aplicación del concepto impone a nuestro razonamiento. De ahí que, como lo ilustra el trabajo de 
Walton, puedan presentarse, más tal vez en unos casos que en otros, dificultades o dudas al momento de formular un esquema de argumentación (y las preguntas críticas que lo acompañan). En nuestra opinión, estas dificultades y dudas podrían superarse de manera más satisfactoria si se reconociera que es esencialmente de la explicitación de las condiciones de aplicación de nuestros conceptos que resulta la formulación de esquemas de argumentación.

Así pues, estas consideraciones muestran que la identificación siempre posible de nuevos esquemas de argumentación, distintos de los que Walton ha podido identificar hasta ahora, más que una dificultad para nuestra propuesta, es algo que nuestra propuesta puede explicar. En las distintas esferas de interacción social en las que existen prácticas argumentativas, distintos conceptos pueden ser empleados. De la explicitación de las condiciones de aplicación de estos conceptos es posible derivar esquemas de argumentación. Si las condiciones de aplicación de los conceptos son correctamente explicitadas, los esquemas resultantes serán racionales. Una persona que posee los conceptos en cuestión, en virtud simplemente de tal posesión, será capaz de captar la racionalidad de dichos esquemas, así como de identificar preguntas críticas que permitan refutar eficazmente su uso en una ocasión particular. Es por ello que podemos sostener que, para todo esquema de argumentación $G$ (presente o futuro), existe al menos un concepto $C$ cuyas condiciones de aplicación explican la racionalidad tanto de $G$ como de las preguntas críticas que lo acompañan y cuya posesión explica nuestra capacidad de captar dicha racionalidad y nuestra capacidad de formular nuevas preguntas críticas.

Dados todos estos planteamientos, es razonable rechazar la idea de que el factor central que explica nuestro dominio de la dimensión lógica de la argumentación es el uso de esquemas como los que formula Walton. Nuestra capacidad de identificar razones relevantes para respaldar o refutar aserciones cuando argumentamos es explicada en virtud simplemente de nuestra capacidad de aplicar conceptos, es decir de nuestra capacidad de formar juicios. Nuestra capacidad de formar juicios no presupone de ningún modo el uso de esquemas de argumentación.

Pero, ¿qué decir de la segunda interpretación de la propuesta de Walton? ¿Los esquemas de argumentación son efectivamente herramientas útiles para analizar y evaluar argumentos y para 
mejorar nuestras prácticas argumentativas? En la medida en que la formulación de esquemas de argumentación no es más que el intento de explicitación de la manera en que las condiciones de aplicación de nuestros conceptos regulan racionalmente nuestros procesos inferenciales, es preciso reconocer la utilidad de dichos esquemas, pues nos permiten ser conscientes, al menos hasta cierto punto, de los mecanismos implícitos que actúan sobre nuestro razonamiento y, como consecuencia de ello, nos permiten también ejercer cierto control reflexivo sobre nuestras prácticas argumentativas. Sin embargo, no por ello debemos asumir, como lo hacen Frans H. Van Eemeren y Francisca Snoeck Henkemans (2017), que, cuando argumentamos, empleamos efectivamente esquemas de argumentación. Van Eemeren y Snoeck Henkemans llegan incluso a afirmar que las razones presentadas para respaldar una determinada aserción no pueden ser consideradas exitosas si no fueron correctamente derivadas del uso de un esquema de argumentación apropiado (Eemeren \& Snoeck, 2017, p. 116). Argumentar de forma existosa no presupone necesariamente el uso correcto de esquemas de argumentación, sino simplemente la sensibilidad a las restricciones o normas racionales que regulan la aplicación de los conceptos que son relevantes en el intercambio argumentativo en el que participamos. Así, hablando de forma estricta, las personas pueden argumentar existosamente sin emplear de ningún modo esquemas de argumentación, contrariamente a lo que suponen Van Eemeren y Snoeck Henkemans.

\section{Conclusión}

En este trabajo, hemos querido mostrar que el factor principal que explica nuestro dominio de la dimensión lógica de la argumentación es nuestra capacidad de formar juicios, una capacidad cognitiva básica de los seres humanos, y no el uso de esquemas de argumentación. Más precisamente, hemos sostenido que es en virtud de nuestra sensibilidad a las condiciones de aplicación de nuestros conceptos que somos capaces de identificar razones que nos permiten eficazmente respaldar nuestras aserciones o refutar las aserciones de nuestro interlocutor en un intercambio argumentativo. Tal sensibilidad es básicamente aquello en lo que consiste poseer un concepto. El proyecto de identificar y formular esquemas de argumentación, junto con sus 
respectivas preguntas críticas, puede ser entendido como un intento de explicitar las normas o restricciones que regulan racionalmente nuestros procesos inferenciales, las cuales derivan esencialmente de las condiciones de aplicación de nuestros conceptos. En la medida en que el intento de explicitación de tales normas o restricciones, a través de la formulación de esquemas de argumentación, es exitoso, el estudio de dichos esquemas puede tener cierta utilidad. Sin embargo, no debemos caer en el error de suponer que las personas, cuando argumentan, emplean realmente esquemas de argumentación. Los temas sobre los cuales podemos argumentar son numerosos y diversos, pero la razón que explica esta absoluta generalidad de la argumentación no es el hecho de que dispongamos de esquemas que, dado precisamente su carácter esquemático, pueden ser empleados en numerosas y diversas ocasiones, sino más bien el hecho de que estos temas no son otros que los numerosos y diversos temas sobre los cuales podemos formar juicios.

\section{Referencias}

Brandom, R. (1994). Making it explicit. Reasoning, representing and discursive commitment. Cambridge, Massachusetts: Harvard University Press.

Brandom, R. (2000). Articulating reasons. An introduction to inferentialism. Cambridge, Massachusetts: Harvard University Press.

Fodor, J. (1990). A theory of content and other essays. Cambridge, Massachusetts: M.I.T. Press. Fodor, J. (1998). Concepts. Where cognitive science went wrong. Oxford: Oxford University Press. Mercier, H. \& Sperber, D. (2017). The enigma of reason. Cambridge, Massachusetts: Harvard University Press.

Millikan, R. G. (2000). On clear and confused ideas. An essay about substance concepts. Cambridge: Cambridge University Press.

Peacocke, C. (1992). A study of concepts. Cambridge, Massachusetts: M.I.T. Press.

Peacocke, C. (1999). Being known. Oxford: Oxford University Press.

Peacocke, C. (2004). The realm of reason. Oxford: Oxford University Press.

Peacocke, C. (2008). Truly understood. Oxford: Oxford University Press. 
Quine, W. V. O. (1953). From a logical point of view. Cambridge, Massachusetts: Harvard University Press.

Eemeren, F. \& Snoeck, F. (2017). Argumentation. Analysis and evaluation. New York: Routledge.

Walton, D. (1996). Argumentation schemes for presumptive reasoning. Mahwah, New Jersey: Erlbaum.

Walton, D. (2013). Methods of argumentation. Cambridge: Cambridge University Press.

Walton, D., Reed, C. \& Macagno, F. (2008). Argumentation schemes. Cambridge: Cambridge University Press.

Wenzel, J. W. (2006). Three perspectives on argument. Rhetoric, dialectic, logic. En R. Trapp \& J. Schuetz (Eds.) Perspectives on argumentation. Essays in honor of Wayne Brockriede (p. 9-26). New York: Idebate Press. 